Authors

Xinye Xu, Thomas H. Loftus, John M. Dunn, Chris H. Greene, John L. Hall, Alan Gallagher, and Jun Ye 


\title{
Single-Stage Sub-Doppler Cooling of Alkaline Earth Atoms
}

\author{
Xinye Xu, Thomas H. Loftus, Josh W. Dunn, Chris H. Greene, John L. Hall, Alan Gallagher, and Jun Ye* \\ JILA, National Institute of Standards and Technology and University of Colorado, and Department of Physics, \\ University of Colorado, Boulder, Colorado 80309-0440 \\ (Received 14 December 2002; revised manuscript received 14 February 2003; published 13 May 2003)
}

We report the first experimental study of sub-Doppler cooling in alkaline earth atoms $\left({ }^{87} \mathrm{Sr}\right)$ enabled by the presence of nuclear spin-originated magnetic degeneracy in the atomic ground state. Sub-Doppler cooling in a $\sigma^{+}-\sigma^{-}$configuration is achieved despite the presence of multiple, closely spaced excited states. This surprising result is confirmed by an expanded multilevel theory of the radiative cooling force. Detailed investigations of system performance have shed new insights into $\left(\sigma^{+}-\sigma^{-}\right)$cooling dynamics and will likely play an important role in the future development of neutral atom-based optical frequency standards.

DOI: 10.1103/PhysRevLett.90.193002

Optical frequency standards based on laser-cooled neutral atoms have been steadily improving in recent years. The demonstrated results are already impressive, from optical standards based on ultranarrow optical transitions, such as the spin-forbidden intercombination lines in even-isotope alkaline earths $[1,2]$. However, for neutral atom-based systems to become truly competitive against, for example, single trapped ions, systematic effects on frequency shifts have to be carefully studied. A crucial step towards ultimate system reproducibility and accuracy will be confining neutral atoms in the LambDicke regime, while at the same time limiting the effect of the confining potential to only the external degrees of freedom without shifting the atomic internal level structure. Precooling alkaline earths to ultralow temperatures is an essential component in these experiments. Furthermore, cooling dynamics in alkaline earth atoms are qualitatively different from the more extensively studied alkali systems. Consequently, interest in various methods for cooling alkaline earth atoms to sub-mK temperatures has received increased attention recently. To date, owing to the lack of ground state electronic structure, obtaining $\sim \mu \mathrm{K}$ temperatures has required second-stage cooling on narrow intercombination lines $[3,4]$.

The fermionic isotopes of alkaline earth atoms, such as ${ }^{87} \mathrm{Sr}$, present an attractive alternative. ${ }^{87} \mathrm{Sr}$ atoms have a nonzero nuclear magnetic moment $(I=9 / 2)$ that gives rise to magnetic substructure in both the ground and excited states. The presence of magnetic degeneracy in the ground state brings an interesting prospect of direct sub-Doppler cooling of ${ }^{87} \mathrm{Sr}$ on the strong ${ }^{1} S_{0^{-}}{ }^{1} P_{1}$ transition, potentially enabling single-step cooling of a large number of atoms to ultralow temperatures. The magnetic degeneracy in the ground state provides sublevel dependent Clebsch-Gordan coefficients that give rise to statedependent level shifts in the presence of appropriately tuned and polarized laser fields. Furthermore, the nuclear spin-based magnetic sensitivity of the ground state sublevels is 1000 times smaller than that of electronically originated structures. Therefore, given an appropriate
PACS numbers: 32.80.Pj, 32.80.Lg, 39.25.+k, 42.50.Vk

excited-state level structure that supports adequate optical pumping, the $\left(\sigma^{+}-\sigma^{-}\right)$sub-Doppler cooling mechanism is expected to work, along with Doppler cooling, even in the presence of reasonably large magnetic field gradients associated with an operating magneto-optic trap (MOT) [5]. This should lead to a direct, single-stage cooling and trapping of a large number of ${ }^{87} \mathrm{Sr}$ atoms with a temperature well below the Doppler limit.

The unique hyperfine structure of the ${ }^{1} P_{1}$ excited state of ${ }^{87} \mathrm{Sr}$ also presents an excellent testing ground for multilevel based sub-Doppler cooling theory [6]. In particular, for systems similar to ${ }^{87} \mathrm{Sr}$, such as $\mathrm{Li}$ and ${ }^{39} \mathrm{~K}$ that have significant spectral overlap in the excited-state manifold, earlier studies have shown a great reduction in the subDoppler cooling force. Indeed, no sub-Doppler cooling has been reported for these systems $[7,8]$. Our observations of sub-Doppler temperatures, along with our theoretical calculations that predict a full scale sub-Doppler cooling force due to the large magnetic degeneracy $(F=$ $9 / 2$ for ${ }^{87} \mathrm{Sr}$ ground state), thus provide a critical insight into the behavior of these complex systems and the nature of the associated sub-Doppler cooling force.

The key feature of ${ }^{87} \mathrm{Sr}$ for the next generation optical frequency standards is the existence of an extremely stable and narrow optical transition, ${ }^{1} S_{0}(F=9 / 2)-{ }_{-}^{3} P_{0}\left(F^{\prime}=9 / 2\right)$, with a linewidth of $\sim 1 \mathrm{mHz}$. This doubly forbidden $J=0$ to $J=0$ transition is expected to be highly insensitive to external electromagnetic fields and collisional shifts. (Note the collisional shift is usually scaled by the transition linewidth and Zeeman shifts are negligibly small for nuclear spinbased magnetic substructure.) When constructing a far-off-resonance lattice trap for these atoms in the Lamb-Dicke regime, a magic wavelength $(\sim 800 \mathrm{~nm})$ will be used so that both the ground and the excited $\left({ }^{3} P_{0}\right)$ states experience the same energy shift anywhere inside the trap [9]. Here the insensitivity to the light polarization of the $J=0$ states will be tremendously useful in practice and of critical importance for measurement accuracy. 
Motivated by these system advantages, we present in this Letter the first detailed measurements of sub-Doppler cooling of ${ }^{87} \mathrm{Sr}$ in a single-stage ${ }^{1} S_{0^{-}}{ }^{1} P_{1}$ MOT. Cooling dynamics associated with trapping beam intensity, detuning, atom density, and the trap loading times are carefully explored. The results demonstrate a MOT temperature for ${ }^{87} \mathrm{Sr}$ that is clearly below the Doppler limit and is strongly dependent on the trapped atom number and cooling beam intensity. From the measured size and temperature of the trapped sample, we determine the associated spring constant $(\kappa)$ and damping coefficient $(\alpha)$, and find they are in good agreement with an expanded multilevel sub-Doppler cooling theory except for a global scaling factor of 10 . Surprisingly, we find that the lack of spectral resolution in the ${ }^{87} \mathrm{Sr}$ excited-state structure does not affect subDoppler cooling dynamics. Our theoretical calculation clearly demonstrates that the magnetic degeneracy plays a more dominant role in the magnitude of sub-Doppler cooling force than the excited-state level spacing.

The atomic energy levels relevant to laser cooling of ${ }^{87} \mathrm{Sr}$ in a ${ }^{1} S_{0^{-}}{ }^{1} P_{1}$ MOT are shown in Fig. 1. Energy spacings between the excited-state hyperfine levels are comparable to the linewidth of the ${ }^{1} S_{0^{-}}{ }^{1} P_{1}$ transition, which has an Einstein $A$ coefficient of $2 \times 10^{8} \mathrm{~s}^{-1}$ [natural linewidth $\Gamma /(2 \pi)=32 \mathrm{MHz}$. The ground state Lande $g$ factor is $\sim 1000$ times smaller than in alkali metal atoms. Thus, polarization-dependent optical pumping through the excited state leads to sub-Doppler cooling, even in the presence of the MOT magnetic field. The experiment consists of a standard six-beam vapor-cell $\mathrm{Sr}$ MOT using the ${ }^{1} S_{0}(F=9 / 2)-{ }^{1} P_{1}\left(F^{\prime}=11 / 2\right)$ transition at $\lambda=461 \mathrm{~nm}$ (resonance frequency $\omega_{0}$ ) [10]. Note that due to the inverted and closely spaced excitedstate structure, the trapping beams also excite the $(F=9 / 2)-\left(F^{\prime}=9 / 2\right)$ and $(F=9 / 2)-\left(F^{\prime}=7 / 2\right)$ transitions. The trapping light detuning, $\left[\delta=\Delta / 2 \pi=\left(\omega_{L}-\right.\right.$ $\left.\left.\omega_{0}\right) / 2 \pi\right]$, is controlled by a double-passed acousto-optic modulator. Similar to ${ }^{88} \mathrm{Sr}$, we find the ${ }^{87} \mathrm{Sr}$ MOT loss rate is primarily determined by radiative branching from the ${ }^{1} P_{1}$ excited-state to the ${ }^{3} P_{2}$ and ${ }^{3} P_{0}$ metastable excited states. For typical trapping beam intensity and detuning, the MOT lifetime is limited to $\sim 20 \mathrm{~ms}$.

Figure 2 shows the measured ${ }^{87} \mathrm{Sr}$ MOT temperature as a function of (a) the total trapping beam intensity at a

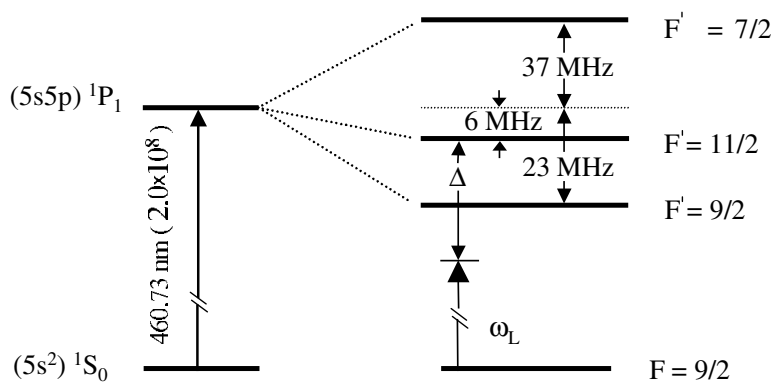

FIG. 1. Energy level structure of the fermionic ${ }^{87} \mathrm{Sr}$ relevant to the ${ }^{1} S_{0^{-}}{ }^{1} P_{1}$ cooling and trapping transition. fixed detuning of $\delta=-40 \mathrm{MHz}$, and (b) the trapping beam detuning at fixed intensities of $I_{t}=48$ and $27 \mathrm{~mW} / \mathrm{cm}^{2}$ [saturation intensity $I_{s}=\pi h c \Gamma /\left(3 \lambda^{3}\right)=$ $43 \mathrm{~mW} / \mathrm{cm}^{2}$ ]. In all cases, the axial magnetic field gradient is $d B_{z}=53 \mathrm{G} / \mathrm{cm}$, and the vapor-cell background pressure, set primarily by thermal $\mathrm{Sr}$, is $\sim 1 \times 10^{-8}$ Torr. For comparison, dotted lines show the temperature dependence expected from Doppler cooling theory. In both plots, sub-Doppler cooling is clearly revealed. Unfortunately the background vapor in our cell limits the lowest number of trapped atoms that can be measured, therefore limiting the lowest temperature reported here. It is worth noting that all previously reported cooling results on even isotopes of alkaline earth atoms have shown temperatures that are a few times higher than the Doppler limit $[10,11]$. We find for ${ }^{88} \mathrm{Sr}$ the Doppler cooling limit is reached only when the cooling beam intensity approaches zero.

To perform these temperature measurements, we use a cloud expansion technique [10,12]. A $200 \mu$ m-diameter, resonant $461 \mathrm{~nm}$ probe beam is sent through the center of the trap, and the absorption of the probe beam is detected by a photodiode. We record decay curves of the sample absorption after the MOT is switched off by turning off both the quadrupole magnetic field and the trapping beams. Note that we do not have a post-MOT subDoppler cooling stage as in usual alkali atom cases: the sub-Doppler cooling is carried out during the MOT stage. Similar to the sub-Doppler cooling work performed on alkali atoms [13], we fit the temperature data in Fig. 2 by $T=a\left(\hbar \Gamma / 2 k_{B}\right)\left(I_{t} / I_{s}\right) /(|\Delta| / \Gamma)+b$, where $a$ is a dimensionless scaling parameter and $b$ signifies the temperature in the zero intensity limit. For Fig. 2(a), a linear fit for intensities $<30 \mathrm{~mW} / \mathrm{cm}^{2}$ gives $a=1.68(0.10)$ and $b=$ $0.17(0.03) \mathrm{mK}$. In Fig. 2(b), we fix the $b$ value at $0.17(0.03) \mathrm{mK}$ and use $a$ as a single fitting parameter. The mean value of $a$ is 1.3(0.3). In the high intensity case, the temperatures rise above Doppler predictions for $|\delta| \leq 40 \mathrm{MHz}$. With the trapping beam intensity at 0.14 of $I_{s}$ and the detuning at $(|\Delta| / \Gamma)=1.25$, the sample temperature is $300 \mu \mathrm{K}$, corresponding to an rms velocity

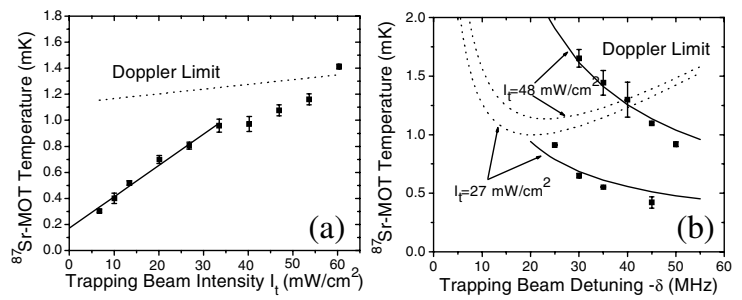

FIG. 2. Dependence of the atomic temperature on (a) the trapping beam intensity at a fixed detuning $\delta=-40 \mathrm{MHz}$, and (b) frequency detuning at two fixed intensities $I_{t}=48$ and $27 \mathrm{~mW} / \mathrm{cm}^{2}$. Fits are shown as solid lines. Dotted lines represent predictions from 1D Doppler cooling theory. 
of $17 \mathrm{~cm} / \mathrm{s}$. Because of the simultaneous presence of the MOT quadrupole magnetic field and the subDoppler cooling force, the captured atom number $\left(>10^{4}\right)$ is significant even for this low intensity regime. Temperatures achieved for ${ }^{87} \mathrm{Sr}$ are significantly lower than that for ${ }^{88} \mathrm{Sr}$ under similar experimental conditions and represent the lowest values for alkaline earth atoms achieved in a single cooling step. The results also represent the first observation of sub-Doppler temperatures in a system with significant spectral overlap in the excitedstate hyperfine manifold.

Because of the unique hyperfine structure of the ${ }^{87} \mathrm{Sr}$ ${ }^{1} P_{1}$ excited state that includes both an inversion (between $F^{\prime}=7 / 2$ and $F^{\prime}=9 / 2$ ) and small energy level spacings, an extended theory of sub-Doppler cooling is required to fully understand ${ }^{87} \mathrm{Sr}$ cooling dynamics $[6,14]$. We have constructed generalized optical Bloch equations to describe interactions between 1D counterpropagating $\sigma^{+}-\sigma^{-}$laser fields and all relevant hyperfine states in ${ }^{87} \mathrm{Sr}$ and their associated Zeeman substates. The high magnetic degeneracy produces a surprising result as shown in Fig. 3, where the relationship between the radiation force and atomic velocity is displayed. First, for comparison, the Doppler cooling force is shown as the dotted line, calculated for the case of $F=0 \rightarrow F=1$, as relevant for ${ }^{88} \mathrm{Sr}$. The radiation force for ${ }^{87} \mathrm{Sr}$ is shown as the solid line, displaying a remarkable sub-Doppler component for atomic speed less than $\sim 0.37 \mathrm{~m} / \mathrm{s}$ ( $0.025 \Gamma / \mathrm{k}$ or a temperature of $1.4 \mathrm{mK}$ ). If we artificially increase the level spacing of the excited-state hyperfine manifold by a factor of 3 , without changing the linewidth or detuning, we find a significantly reduced sub-Doppler cooling force as depicted by the dashed line in the figure. However, if the energy spacing is further increased, for example, at 5 times the original value, we find the cooling force is basically the same as that for the original level spacing and can be represented by the same solid curve in the figure. Reducing the energy spacing by a factor of 2 also preserves the full scale of the cooling force.

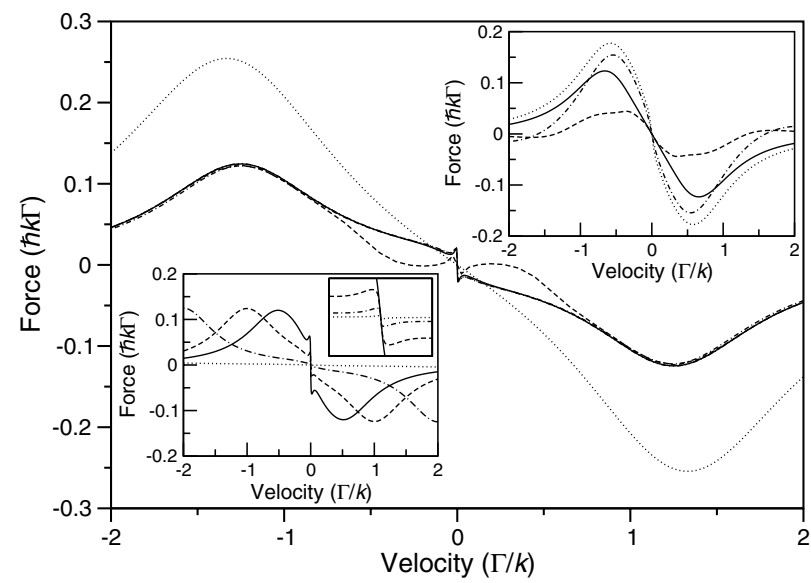

FIG. 3. Calculated radiation cooling force vs atomic velocity. See text for details. $k$ : light wave vector.
Further calculations on an isolated transition $(F=9 / 2$ to $\left.F^{\prime}=11 / 2\right)$, as well as on $\left(F=4\right.$ to $\left.F^{\prime}=5\right)$ for Cs atoms, reveal very similar results. A full scale subDoppler cooling force thus exists for ${ }^{87} \mathrm{Sr}$ atoms. All calculations are performed at a total intensity $\sim I_{s}$ and detuning $\sim-\Gamma$.

These results are in sharp contrast to those obtained for low magnetic degeneracy [6-8], where small level spacings in the excited-state manifold are responsible for the lack of sub-Doppler cooling dynamics. (Another reason for the lack of sub-Doppler cooling dynamics in Li or $\mathrm{K}$ MOTs could be that for an already weakened sub-Doppler cooling force, the requirement of zero magnetic field is 1000 times more stringent than for Sr.) For a clear comparison, we have performed calculations for the case of $F=1$ to $F^{\prime}=0,1,2$. The level spacing between $F^{\prime}=0$ and $1\left(F^{\prime}=1\right.$ and 2$)$ is first assumed to be $0.15(0.4)$ of the transition linewidth, and is then progressively increased by 5,20 , and 100 times, with a fixed laser detuning from $F^{\prime}=2$. Results on these four cases are shown, respectively, as the solid, dashed, dot-dashed, and dotted curves in the upper-right inset of Fig. 3. Similar to ${ }^{87} \mathrm{Sr}$, we again notice an interesting resonancelike dip in the radiation force when the energy spacings are (artificially) increased to a region where the laser is tuned near resonance on the blue side of the lower hyperfine state. Nevertheless, it is clear that small energy spacings coupled with low magnetic degeneracy greatly reduces the sub-Doppler cooling force. In the lower-left inset of Fig. 3 we show the dependence of the sub-Doppler cooling force on the frequency detuning of the cooling beam with respect to the ${ }^{87} \mathrm{Sr}^{1} P_{1}$ $F^{\prime}=11 / 2$ state. When the detuning is increased from $-0.5 \Gamma$ to $-1 \Gamma,-2 \Gamma$, and then $-5 \Gamma$, the associated cooling force decreases rapidly, with results shown, respectively, in the solid, dashed, dot-dashed, and dotted curves. The inset to this inset shows an expanded $(5 \times$ vertical, $40 \times$ horizontal) view of the central region. Our experimental choice of detuning, $-1.25 \Gamma(-40 \mathrm{MHz})$, represents the best compromise between cooling and heating mechanisms.

Based on the equipartition theorem, $\frac{1}{2} \kappa r_{\text {rms }}^{2}=\frac{1}{2} k_{B} T$, we can determine the trap spring constant $(\kappa)$ from the measured temperature and the trapped sample size $\left(r_{\text {rms }}\right)$. The damping coefficient $\alpha$ can be determined from the relation $\alpha=\kappa /\left[d B_{z} g_{F} \mu_{B} /(\hbar k)\right]$ [15], where $g_{F}=2 / 11$ is the Lande $g$ factor of the ${ }^{1} P_{1}\left(F^{\prime}=11 / 2\right)$ excited state, $\mu_{B}$ is the Bohr magneton, and $k=2 \pi / \lambda$ is the laser wave vector. Figure 4 shows the measured (in filled squares) dependence of the damping coefficient (left vertical axis) and the spring constant (right vertical axis) on the trapping beam intensity. The theoretical curve (in open squares) is produced by the ratio of force to velocity evaluated at the rms velocity. The agreement between theory and experiment on the intensity dependence is good, after the theory data are divided by 10 . The same scaling discrepancy has been observed with experimental results on alkali atoms [15]. 


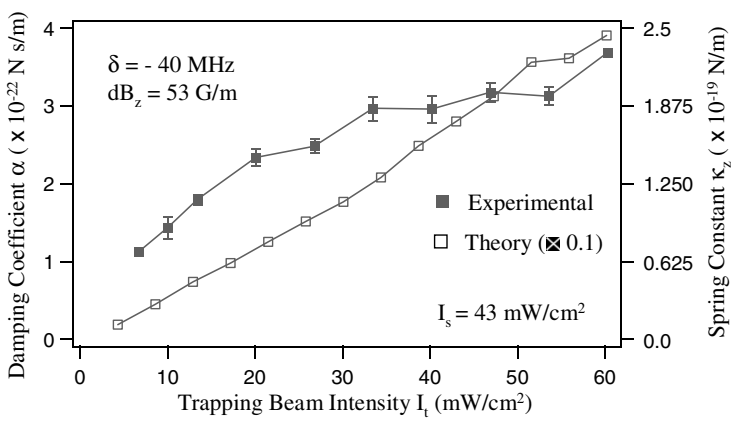

FIG. 4. Dependence of the damping coefficient (left axis) and the spring constant (right axis) on the trapping beam intensity at a fixed detuning $\delta=-40 \mathrm{MHz}$. Theory curve is overlaid with a scaling factor of 0.1 .

The remaining question for the ${ }^{87} \mathrm{Sr}$ system is that the cooling temperature is higher than what should be expected from a sub-Doppler cooling force comparable to that for Cs atoms. Under similar experimental conditions, the momentum diffusion coefficient for ${ }^{87} \mathrm{Sr}$ is only $\sim 3$ times larger than for $\mathrm{Cs}$, after normalization to $\Gamma$. To explore these issues and in accordance with previous studies of radiation trapping in cases of varying sample density [14], we have measured the sample temperature with respect to the number and density $\left(n^{2 / 3} N^{1 / 3}\right)$. We vary these two parameters using several different techniques. In the first approach, we maintain $I_{t}=$ $14 \mathrm{~mW} / \mathrm{cm}^{2}, \delta=-40 \mathrm{MHz}$, and $d B_{z}=53 \mathrm{G} / \mathrm{cm}$ fixed and simply change the background $\mathrm{Sr}$ partial pressure (triangles). The measured slope, $\Delta T / \Delta\left(n^{2 / 3} N^{1 / 3}\right)$, is $0.342(0.002) \times 10^{-8} \mathrm{mK} / \mathrm{cm}^{2}$. In the second approach, we make a sudden switch in the trapping beam intensity from $I_{t}=32 \mathrm{~mW} / \mathrm{cm}^{2}$ to $I_{t}=14 \mathrm{~mW} / \mathrm{cm}^{2}$, and monitor the subsequent decay of the sample number and density, along with the sample temperature, before they reach a new equilibrium (circles). The resultant $\Delta T / \Delta\left(n^{2 / 3} N^{1 / 3}\right)$ is similar to the first case, at $0.274(0.058) \times$ $10^{-8} \mathrm{mK} / \mathrm{cm}^{2}$. The slope of our measurement is approximately twice the predicted value based on radiation trapping [14]. However, we note that the prediction was derived from previous alkali atom experiments where the trapping beam intensity was changed. Hence, in the third approach, we measure the number and density dependence of the trap temperature by changing the steadystate trapping beam intensity (diamond). The resultant $\Delta T / \Delta\left(n^{2 / 3} N^{1 / 3}\right)$ is more than 15 times bigger than the previous two cases, $\sim 4.85(0.41) \times 10^{-8} \mathrm{mK} / \mathrm{cm}^{2}$. The alkaline earth atoms apparently have an extra heating mechanism that is highly sensitive (with a quadratic dependence) to the light intensity. We note in passing that for the current experiment, the density for the ${ }^{3} P_{2}$ state is less than $10^{6} \mathrm{~cm}^{-3}$ and the nonresonant collision rate between ${ }^{1} S_{0}$ and ${ }^{3} P_{2}$ atoms is less than $10^{-11} \mathrm{~cm}^{3} / \mathrm{s}$. Thus the collision loss mechanism provides negligible heating effects.

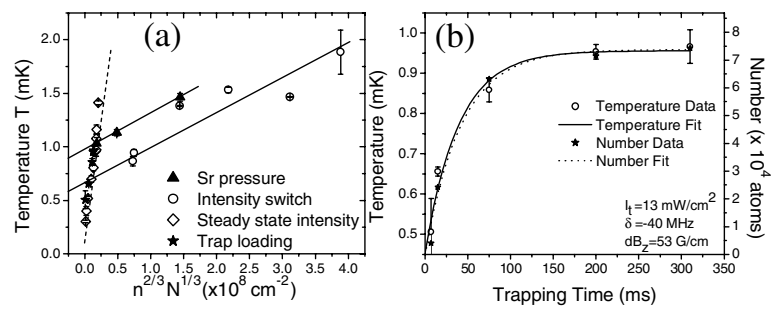

FIG. 5. Dependence of the temperature on (a) the trap number and density (see text for details) and (b) the trap loading time. In (a), the solid lines are fits that we compare against radiation trapping theory. The dashed line is a visual guide. In (b), both the solid and dotted lines are fits.

Finally we present an interesting case where the trap loading time is varied and we monitor the trap number and density along with the corresponding temperature at the end of loading (star). We find that the trap temperature exponentially grows with a $1 / e$ time of $40 \mathrm{~ms}$, the same as the trap number growth, as shown in Fig. 5(b). The temperature dependence on the number and density is also relatively large, with $\Delta T / \Delta\left(n^{2 / 3} N^{1 / 3}\right) \sim$ $3.74(0.21) \times 10^{-8} \mathrm{mK} / \mathrm{cm}^{2}$. This effect is attributed to the fact that hot atoms loaded into the trap take a few tens of milliseconds to reach the equilibrium temperature, although the dynamic time scale for an equilibrated trap is short, at $\sim 2 \mathrm{~ms}$.

We acknowledge contributions from M. Boyd and T. Ido. This work is funded by ONR, NSF, NASA, and NIST. T. Loftus was supported by NRC.

*Corresponding author.

Email address: Ye@jila.colorado.edu

[1] F. Riehle et al., IEEE Trans. Instrum. Meas. 48, 613 (1999).

[2] T. Udem et al., Phys. Rev. Lett. 86, 4996 (2001).

[3] H. Katori et al., Phys. Rev. Lett. 82, 1116 (1999).

[4] T. Binnewies et al., Phys. Rev. Lett. 87, 123002 (2001).

[5] S. Chu, Rev. Mod. Phys. 70, 685 (1998); C. CohenTannoudji, ibid. 70, 707 (1998); W. D. Phillips, ibid. 70, 721 (1998).

[6] A. Bambini and A. Agresti, Phys. Rev. A 56, 3040 (1997).

[7] C. Fort et al., Eur. Phys. J. D 3, 113 (1998).

[8] U. Schunemann et al., Opt. Commun. 158, 263 (1998).

[9] H. Katori, in Proceedings of the 6th Symposium on Frequency Standards \& Metrology, edited by P. Gill (World Scientific, Singapore, 2002), p. 323.

[10] X. Xu et al., Phys. Rev. A 66, 011401(R) (2002).

[11] T. Kisters et al., Appl. Phys. B 59, 89 (1994).

[12] D. S. Weiss et al., J. Opt. Soc. Am. B 6, 2072 (1989).

[13] C. Salomon et al., Europhys. Lett. 12, 683 (1990).

[14] C. J. Cooper et al., Europhys. Lett. 28, 397 (1994).

[15] C. D. Wallace et al., J. Opt. Soc. Am. B 11, 703 (1994). 